\section{BRAZIULIAN JOURNAL \\ OF MEDICAL AND BIOLOGICAL RESF.ARCH}

www.bjournal.com.br
ISSN 0100-879X

Volume 42 (10) 870-992 October 2009

BIOMEDICAL SCIENCES

AND

CLINICAL INVESTIGATION

Braz J Med Biol Res, October 2009, Volume 42(10) 921-929

Hind limb ischemic preconditioning induces an anti-inflammatory response by remote organs in rats.

M.V.P. Souza Filho, R.T. Loiola, E.L. Rocha, A.F.L. Simão, A.S. Gomes, M.H.L.P. Souza and R.A. Ribeiro

The Brazilian Journal of Medical and Biological Research is partially financed by
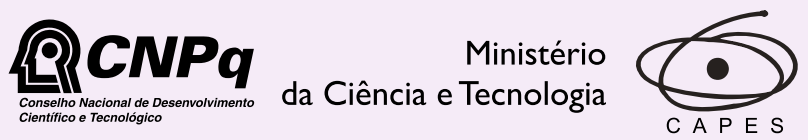

Ministério da Educação
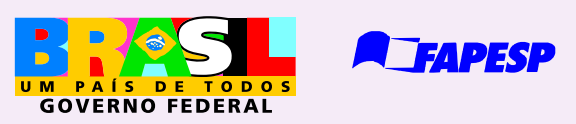

Institutional Sponsors 


\title{
Hind limb ischemic preconditioning induces an anti-inflammatory response by remote organs in rats
}

\author{
M.V.P. Souza Filho ${ }^{1,3}$, R.T. Loiola², E.L. Rocha², A.F.L. Simão², \\ A.S. Gomes ${ }^{2}$, M.H.L.P. Souza ${ }^{2}$ and R.A. Ribeiro ${ }^{1,2,3}$ \\ ${ }^{1}$ Departamento de Cirurgia, ${ }^{2}$ Departamento de Fisiologia e Farmacologia, \\ Faculdade de Medicina, Universidade Federal do Ceará, Fortaleza, CE, Brasil \\ ${ }^{3}$ Instituto do Câncer do Ceará, Fortaleza, CE, Brasil
}

\begin{abstract}
Ischemic preconditioning (IPC), a strategy used to attenuate ischemia-reperfusion injury, consists of brief ischemic periods, each followed by reperfusion, prior to a sustained ischemic insult. The purpose of the present study was to evaluate the local and systemic anti-inflammatory effects of hind limb IPC in male Wistar rat (200-250 g) models of acute inflammation. IPC was induced with right hind limb ischemia for $10 \mathrm{~min}$ by placing an elastic rubber band tourniquet on the proximal part of the limb followed by 30 min of reperfusion. Groups $(\mathrm{N}=6-8)$ were submitted to right or left paw edema (PE) with carrageenan (100 $\mu \mathrm{g})$ or Dextran $(200 \mu \mathrm{g})$, hemorrhagic cystitis with ifosfamide $(200 \mathrm{mg} / \mathrm{kg}$, ip) or gastric injury $(\mathrm{Gl})$ with indomethacin $(20 \mathrm{mg} / \mathrm{kg}, \mathrm{vo})$. Controls received similar treatments, without IPC (Sham-IPC). PE is reported as variation of paw volume (mL), vesical edema $(\mathrm{VE})$ as vesical wet weight $(\mathrm{mg})$, vascular permeability $(\mathrm{VP})$ with Evans blue extravasation $(\mu \mathrm{g}), \mathrm{GI}$ with the gastric lesion index (GLI; total length of all erosions, $\mathrm{mm}$ ), and neutrophil migration (NM) from myeloperoxidase activity. The statistical significance $(P<0.05)$ was determined by ANOVA, followed by the Tukey test. Carrageenan or Dextran-induced PE and VP in either paw were reduced by IPC (42-58.7\%). IPC inhibited VE (38.8\%) and VP (54\%) in ifosfamide-induced hemorrhagic cystitis. GI and NM induced by indomethacin were inhibited by IPC (GLI: $90.3 \%$; NM: 64\%). This study shows for the first time that IPC produces local and systemic anti-inflammatory effects in models of acute inflammation other than ischemia-reperfusion injury.
\end{abstract}

Key words: Ischemic preconditioning; Carrageenan; Dextran; Ifosfamide; Indomethacin

\section{Introduction}

The restoration of blood supply to organs after a certain period of no flow ischemia results in parenchymal damage referred to as ischemia-reperfusion injury (IRI). The critical ischemia period in humans depends on the organ and is 15$20 \mathrm{~min}$ in the liver and kidney and $2.5 \mathrm{~h}$ in skeletal muscle, whereas in the brain ischemia for more than 5 min leads to considerable neuronal death and infarction. Reperfusion after periods exceeding the critical ischemia period results in endothelial and parenchymal injury (1).

IRI may affect a number of organs and tissues during blood flow restoration after a prolonged ischemic insult and can be deleterious in various clinical settings due to complications caused by delayed vascular flow recovery. $I R I$, if severe, can offset the benefits of procedures such as organ transplantation, free flap reconstruction, transluminal angioplasty, and coronary artery bypass surgery (2).

Following a period of ischemia, tissues adapt to anaerobic metabolism. Restoration of blood supply results in oxygen supply in excess of the requirements that lead to activation of macrophages in the vasculature and consequently generation of super oxide radicals, also referred to as reactive oxygen species (ROS), causing oxidative stress. The key event in the initial phase of reperfusion injury is activation of macrophages that are the primary source of extracellular ROS. ROS are the key initiators of reperfusion injury, which leads to endothelial injury and further release of pro-inflammatory cytokines (1).

ROS have been implicated in both recruitment and

Correspondence: M.V.P. Souza Filho, Departamento de Fisiologia e Farmacologia, Faculdade de Medicina, Universidade Federal do Ceará, Rua Cel. Nunes de Melo, 1127, 60431-970 Fortaleza, CE, Brasil. Fax: +55-85-288-8333. E-mail: mvponte@ig.com.br

Research supported by CNPq and CAPES.

Received January 16, 2009. Accepted August 10, 2009. Available online September 11, 2009. 
activation of leukocytes in IR as well as IRI, on the basis of evidence that scavenging of these radicals inhibits leukocyte adhesion, infiltration, and the onset of tissue injury. Support for the chain of events implicating ROS in IRI stems from in vitro and in vivo experiments in which application of specific ROS inhibitors attenuated IRI. However, many sources of ROS probably exist. For example, ROS are produced by leukocytes, mast cells, capillary endothelial cells, and muscle cells. Many of the intracellular enzymes involved, including xanthine oxidase, have been identified in several different IRI models. In models of short-term skeletal muscle IRI, the endothelial cell xanthine oxidase, rather than plugging or adherent leukocytes, is primarily responsible for the tissue damage observed after short-term IRI (3).

Ischemic preconditioning (IPC) is the most powerful innate mechanism protecting against IRI. The effects of IPC have been observed in a number of mammalian species, including humans. The method consists of inducing brief periods of sublethal local tissue ischemia as a protection against subsequent lethal ischemia. The early phase of IPC, referred to as "classic preconditioning", is observed immediately after brief ischemia and lasts approximately 3 h. A late phase ("second window") of preconditioning has also been demonstrated 18-24 h after induction of brief ischemia (4).

A more intriguing form of IPC with potentially greater clinical significance is the so-called "remote IPC" (rIPC). Transient tissue ischemia at a remote site can confer subsequent protection of organs exposed to potentially lethal ischemia. The magnitude of its protection was equivalent to that of local IPC (5).

In direct IPC, the early phase of protection is protein synthesis independent and this continues into a later phase of protection, which is protein synthesis dependent. Similarly, the protection from rIPC in the early stage of reperfusion injury is carried into a delayed phase of the injury with many studies confirming the existence of two phases of protection. The delayed phase of rIPC seems to be dependent on protein synthesis. Limb rIPC protected the cerebral endothelium of rats in both the early $(15 \mathrm{~min})$ and late (48 h) phase of reperfusion injury (6).

Recently, an early and late phase of remote protection of the endothelium has been shown in humans with protection being activated immediately after rIPC and lasting $4 \mathrm{~h}$. A delayed phase of protection beginning at $24 \mathrm{~h}$ and lasting $48 \mathrm{~h}$ was noted and was associated with protein expression in the vascular endothelium (6).

Although the precise molecular mechanism of IPC remains unclear, several pathways have been suggested. These include activation of $G$ protein-coupled receptors, protein kinase $\mathrm{C}$, mitogen-activated protein kinases, ATPsensitive $\mathrm{K}^{+}$channels, heat shock protein, adenosine, metal ions, oxygen and nitrogen reactive species, oxidized and biological active lipids, cytokines, catecholamines, and nitric oxide (NO) synthase $(7,8)$.
Although there is substantial evidence to suggest that the mechanisms of IPC converge on the mitochondrial ATPsensitive $\mathrm{K}^{+}$channels, it is becoming apparent that IPC also involves changes in gene expression (9). In fact, rIPC has recently been shown to lead to changes in inflammatory gene expression in circulating human leukocytes (10).

A number of studies have tested the protective effect of IPC on local (11) and systemic (12) IRI-induced inflammation. However, to our knowledge, no study has so far tested the effect of IPC on other models of acute inflammation. The present study was therefore designed to evaluate the local and systemic effects of hind limb IPC on rat models of acute inflammation in the form of carrageenan $(\mathrm{Cg})$ - or Dextran (Dx)-induced right or left hind paw edema, ifosfamide (IFO)-induced hemorrhagic cystitis ( $\mathrm{HC})$, and indomethacin (INDO)-induced gastric injury.

\section{Material and Methods}

\section{Animals}

Male Wistar rats (200-250 g) were housed in temperature-controlled rooms and received water and food ad libitum. The ethical guidelines described in the National Institutes of Health Guide for Care and Use of Laboratory Animals were followed throughout the experiments, with approval of the Committee of Ethics in Animal Research and Care of the Federal University of Ceará.

\section{Drugs}

The following drugs were used: carrageenan (Biochemical Products, USA), Dextran 70 (Pharmacia/Pfizer, Brazil), Evans blue (Sigma, USA), ifosfamide (Holoxane: ASTA Médica-AG, Germany), indomethacin (Prodome Química e Farmacêutica, Brazil), and Tris buffer (Merck, Brazil). All other chemicals were of analytical grade or equivalent.

\section{Ischemic preconditioning protocol}

The IPC protocol was similar to that used by Küntscher et al. (13) with few modifications. Ischemia was induced in the right hind limb by placing an elastic rubber band tourniquet on the proximal part of the limb for $10 \mathrm{~min}$. The limb was then reperfused for $30 \mathrm{~min}$, followed by an inflammatory challenge. Ischemia was monitored with Evans blue dye (25 mg/kg, iv) administered immediately after tourniquet ischemia induction. The dye was not visible in the limb during ischemia, but became visible after tourniquet release.

\section{Hind paw edema}

Paw edema was induced by subplantar injection of $\mathrm{Cg}(100 \mu \mathrm{g} / \mathrm{paw})$ or Dx $(200 \mu \mathrm{g} / \mathrm{paw})$ in a final volume of $0.1 \mathrm{~mL}$. All drugs were dissolved in sterile saline. Control animals received sterile saline only $(0.1 \mathrm{~mL} / \mathrm{paw})$. Hind paw edema was quantified by changes in paw volume or vascular permeability. Paw volume was measured with a hydroplethysmometer (Ugo Basile 7140, Plethysmometer, 
Italy) immediately before treatment and 2, 4, 8, and $24 \mathrm{~h}$ after $\mathrm{Cg}$ administration or $30 \mathrm{~min}, 1,2,4$, and $6 \mathrm{~h}$ after challenge with Dx. The increase in paw volume $(\mathrm{mL})$ was obtained by subtracting the paw volume measured before stimulus injection (14) and was reported as means \pm SEM. Paw vascular permeability was quantified by the Evans blue dye extravasation technique (15). Evans blue (25 mg/kg) was administered intravenously via the penial venous sinus $1 \mathrm{~h}$ before the animals were sacrificed. The paws were then removed, placed in glass tubes containing $4 \mathrm{~mL}$ formamide and incubated overnight at $56^{\circ} \mathrm{C}$. The absorbance of the extracted dye was measured at $630 \mathrm{~nm}$ and the results are reported as means \pm SEM of Evans blue $\mu \mathrm{g} / \mathrm{g}$ paw weight.

\section{Hemorrhagic cystitis model}

IFO $(200 \mathrm{mg} / \mathrm{kg})$ was injected ip and the animals were sacrificed by cervical dislocation $24 \mathrm{~h}$ later. Control animals received sterile saline only. The bladder was then removed by abdominal incision and emptied of urinary contents. A preliminary study on cyclophosphamide-induced HC (16) showed that, based on bladder wet weight, the best time to assess $\mathrm{HC}$ in rats is $24-48 \mathrm{~h}$ after stimulation. After this period the bladder wet weight drops significantly. Therefore, in the present study, we chose to assess IFO-induced HC at $24 \mathrm{~h}(17)$. The quantification of vesical edema was based on changes in bladder wet weight $(18,19)$ and vascular permeability (20). Bladder wet weight is reported in $\mathrm{mg}$ as means \pm SEM. Vesical vascular permeability was quantified by the Evans blue dye extravasation technique. The dye $(25 \mathrm{mg} / \mathrm{kg}$ ) was administered intravenously via the penial venous sinus $1 \mathrm{~h}$ before the animals were sacrificed. The bladders were then dissected, placed in glass tubes containing $1.5 \mathrm{~mL}$ formamide and incubated overnight at $56^{\circ} \mathrm{C}$. The absorbance of the extracted dye was measured at $630 \mathrm{~nm}$ and the results are reported as means \pm SEM of Evans blue, $\mu \mathrm{g} / \mathrm{g}$ bladder weight.

\section{Gastric injury model}

Gastric injury was induced by intragastric instillation of INDO $(20 \mathrm{mg} / \mathrm{kg})$ dissolved in Tris buffer $(1.22 \%$ hydroxymethyl aminomethane in distilled water, $1 \mathrm{~mol} / \mathrm{L}$, w/v, $\mathrm{pH}$ 8.0). Control animals received vehicle (Tris buffer) only. The rats were euthanized $3 \mathrm{~h}$ after stimulation by cervical dislocation. The stomach was immediately removed, opened with an incision along the greater curvature and pinned out on a wax platform. Hemorrhagic or ulcerative lesions were counted and their length was measured with a digital caliper under a magnifying lens. The gastric damage score (gastric lesion index) corresponded to the sum of the lengths ( $\mathrm{mm}$ ) of all linear lesions (21) measured by an observer (ASG) blinded to the treatment regimen. Full-thickness samples of the gastric corpus were then weighed, frozen and stored at $-70^{\circ} \mathrm{C}$ for subsequent myeloperoxidase (MPO) activity assay. MPO is found primarily in the azurophilic granules of neutrophils and therefore has been used extensively as a biochemical marker of granulocyte infiltration into various tissues, including the gastrointestinal tract. The extent of neutrophil accumulation in the gastric mucosa was measured by MPO activity assay, as described in Ref. 22. Briefly, 50-100 mg gastric tissue was homogenized in $1 \mathrm{~mL}$ potassium buffer with $0.5 \%$ hexadecyltrimethylammonium bromide for each $50 \mathrm{mg}$ tissue. The homogenate was then centrifuged at $40,000 \mathrm{~g}$ for $7 \mathrm{~min}$ at $4^{\circ} \mathrm{C}$. MPO activity in the resuspended pellet was assayed by measuring the change in absorbance at $450 \mathrm{~nm}$ using o-dianisidine dihydrochloride and $1 \%$ hydrogen peroxide. The results are reported as MPO units/mg tissue. One MPO unit corresponds to the amount of activity capable of converting $1 \mu \mathrm{mol}$ hydrogen peroxide to water in $1 \mathrm{~min}$ at $22^{\circ} \mathrm{C}$.

\section{Statistical analysis}

Data are reported as means \pm SEM and were analyzed statistically by analysis of variance (ANOVA) followed by the Tukey test, with the level of significance set at $P<0.05$.

\section{Results}

\section{Hind paw edema}

Subplantar injection of $\mathrm{Cg}$ in the right paw induced a progressive increase in paw volume, which peaked at $4 \mathrm{~h}$. However, this increase was significantly reduced by IPC of the right hind limb (Figure 1A). When induced by Dx, the onset was faster and the increase was greater, peaking at $1 \mathrm{~h}$ and remaining elevated up to $4 \mathrm{~h}$ after induction. This increase was also significantly reduced by IPC of the right hind limb (Figure 1B).

Subplantar injection of $\mathrm{Cg}$ in the right hind paw induced a significant increase in Evans blue dye extravasation after $4 \mathrm{~h}$. This increase was significantly reduced by IPC of the limb. Dx induced an even greater increase in Evans blue dye extravasation at $1 \mathrm{~h}$, which was also significantly reduced by IPC (Figure 2).

Ischemic preconditioning of the right hind limb significantly reduced the $\mathrm{Cg}$-induced increase in left paw volume at $4 \mathrm{~h}$. A similar effect was observed at $1 \mathrm{~h}$ when Dx was substituted for $\mathrm{Cg}$ (Figure 3).

The increase in Evans blue extravasation induced $4 \mathrm{~h}$ after subplantar injection of carrageenan into the left hind paw was significantly inhibited when animals were submitted to right hind limb IPC. The Dx-induced increase in Evans blue extravasation in the left paw at $1 \mathrm{~h}$ was also reduced by right hind limb IPC (Figure 4).

\section{Hemorrhagic cystitis}

Cystitis observed $24 \mathrm{~h}$ after IFO administration was characterized macroscopically by the presence of severe edema, hyperemia and marked hemorrhage with mucosal hematomas and intravesical clots. These changes were attenuated by IPC in the right hind limb. 
The IFO-induced increase in bladder wet weight was significantly reduced by IPC of the right hind limb (Figure 5A). Similar effects were observed for the IFO-induced increase in vesical Evans blue dye extravasation (Figure 5B).

\section{Gastric injury}

Three hours after INDO administration, severe hyperemia and hemorrhagic and ulcerative lesions were observed on the gastric mucosa of the animals. These changes were
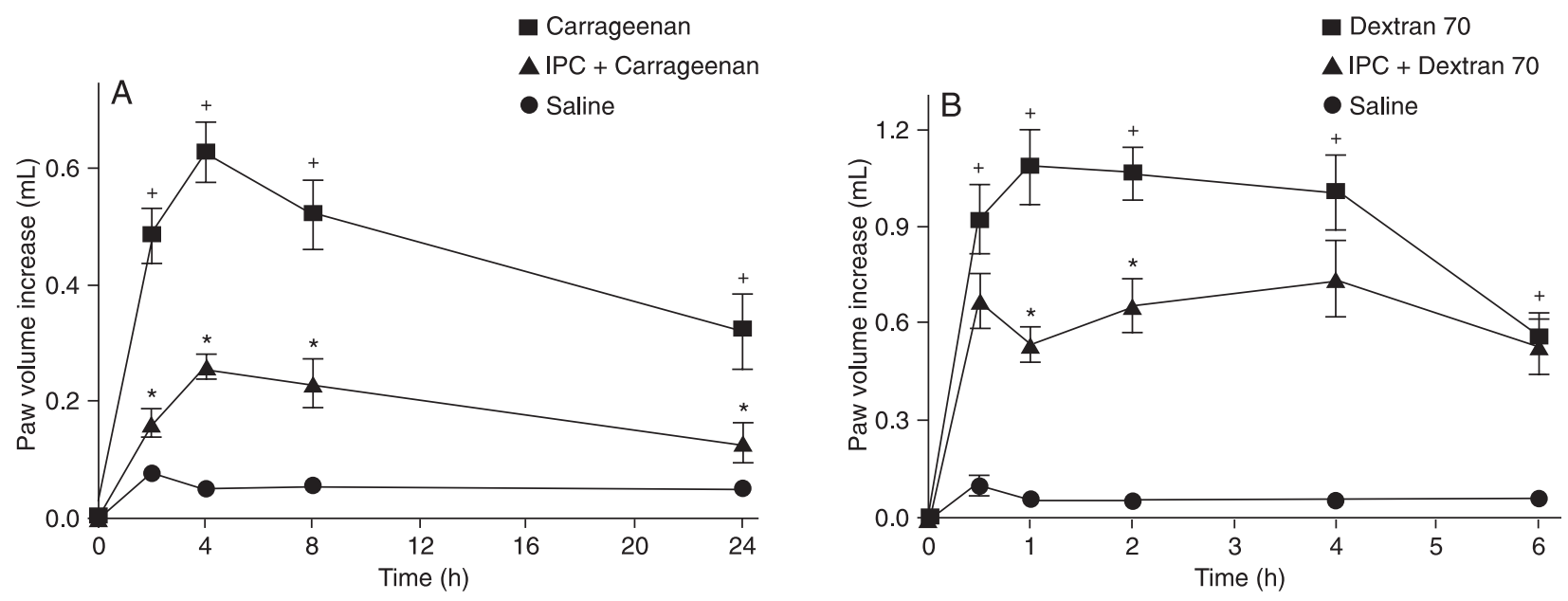

Figure 1. Effect of ischemic preconditioning on ipsilateral rat paw edema induced by carrageenan (A) or Dextran 70 (B). $A$, Edema was measured after carrageenan administration. The animals in the carrageenan group received carrageenan without ischemic preconditioning (IPC). The animals in the saline group received only saline $(0.1 \mathrm{~mL})$. B, Edema was measured after Dextran 70 administration The animals in the Dextran 70 group received Dextran 70 without IPC. The animals in the saline group received only saline $(0.1 \mathrm{~mL})$. Data are reported as means \pm SEM. ${ }^{*} \mathrm{P}<0.05$ compared to carrageenan or Dextran $70{ }^{+} \mathrm{P}<0.05$ compared to saline (ANOVA, followed by the Tukey test).

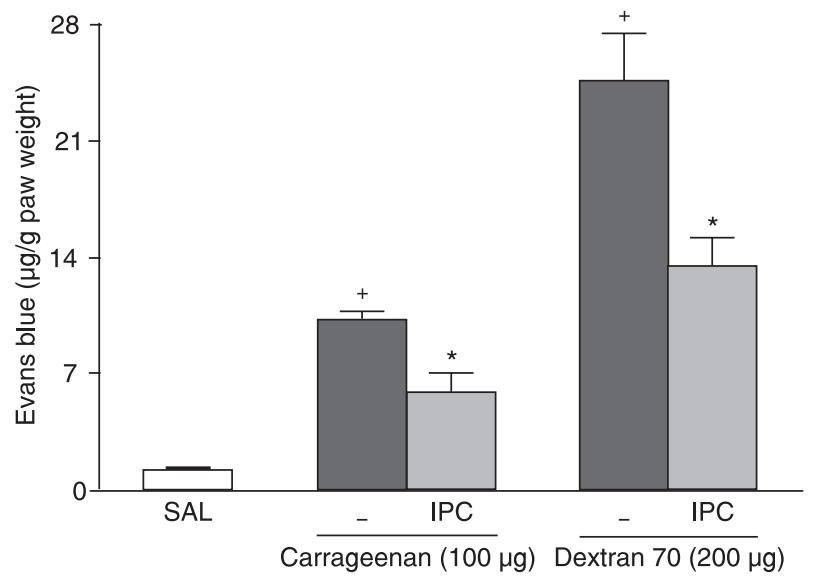

Figure 2. Effect of ischemic preconditioning on the increase in ipsilateral rat paw vascular permeability induced by carrageenan or Dextran 70. The column heights indicate the Evans blue extravasation after saline (SAL), after only carrageenan or Dextran $70(-)$, and after carrageenan or Dextran 70 plus ischemic preconditioning (IPC). Data are reported as means \pm SEM. ${ }^{*} \mathrm{P}<0.05$ compared to carrageenan or Dextran $70 ;{ }^{+} \mathrm{P}<0.05$ compared to saline (ANOVA, followed by the Tukey test).

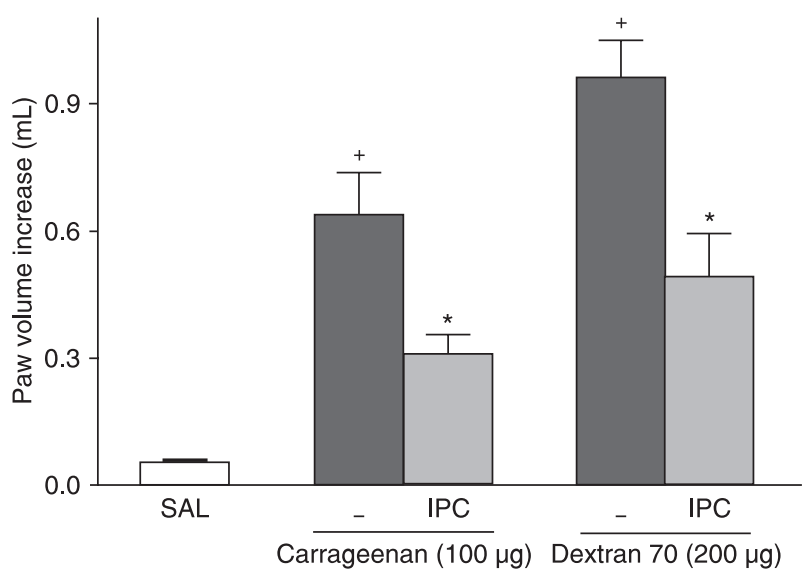

Figure 3. Effect of rat hind limb ischemic preconditioning on contralateral paw edema induced by carrageenan or Dextran 70. The column heights indicate the paw volume increase after saline (SAL), after only carrageenan or Dextran $70(-)$, and after carrageenan or Dextran 70 plus ischemic preconditioning (IPC). Data are reported as means \pm SEM. ${ }^{*} \mathrm{P}<0.05$ compared to carrageenan or Dextran $70{ }^{+} \mathrm{P}<0.05$ compared to saline (ANOVA, followed by the Tukey test). 
visibly attenuated by IPC of the right hind limb (Figure 6). Intragastric instillation of INDO (20 mg/kg) caused sig-
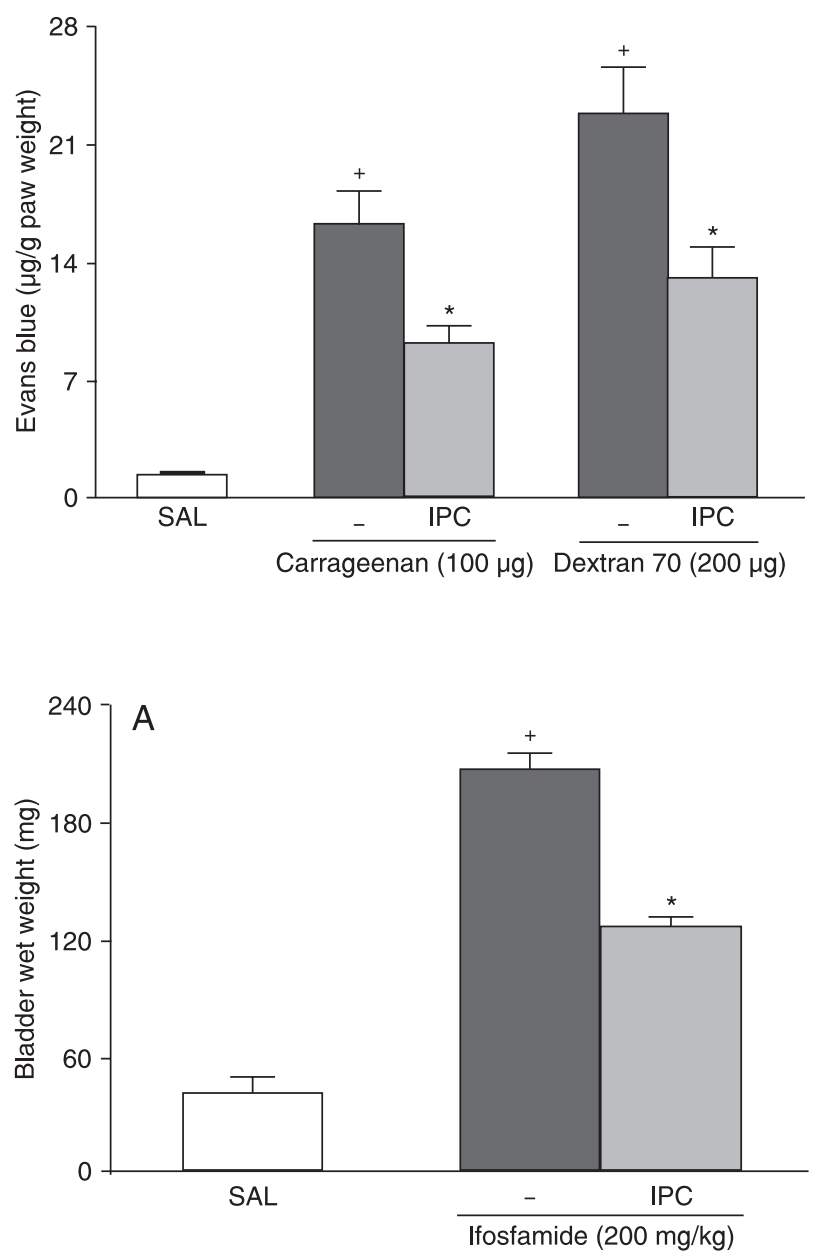

nificant gastric injury after $3 \mathrm{~h}$. This injury was significantly inhibited by IPC of the right hind limb (Figure 7A). Figure

Figure 4. Effect of rat hind limb ischemic preconditioning on the increase of vascular permeability in the contralateral paw induced by carrageenan or Dextran 70 . The column heights indicate the Evans blue extravasation after saline (SAL), after only carrageenan or Dextran 70 (-), and after carrageenan or Dextran 70 plus ischemic preconditioning (IPC). Data are reported as means \pm SEM. ${ }^{*} \mathrm{P}<0.05$ compared to carrageenan or Dextran $70 ;{ }^{+} \mathrm{P}<$ 0.05 compared to saline (ANOVA, followed by the Tukey test).

Figure 5. Effect of rat hind limb ischemic preconditioning on vesical edema (A) and vesical increase of vascular permeability (B) induced by ifosfamide. $A$, The column heights indicate the bladder wet weight after saline (SAL), after only ifosfamide (-), and after ifosfamide plus ischemic preconditioning (IPC). $B$, The columns indicate the vesical Evans blue extravasation after saline (SAL), after only ifosfamide (-), and after ifosfamide plus ischemic preconditioning (IPC). Data are reported as means \pm SEM. ${ }^{*} \mathrm{P}<0.05$ compared to ifosfamide; ${ }^{+} \mathrm{P}<0.05$ compared to saline (ANOVA, followed by the Tukey test).
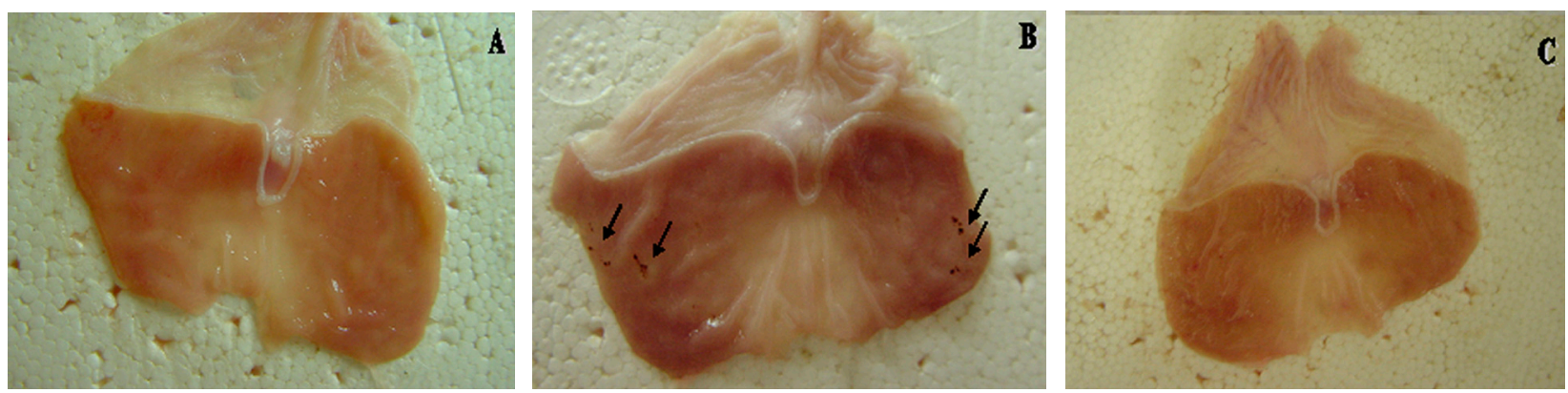

Figure 6. Macroscopic aspect of rat stomachs $3 \mathrm{~h}$ after intragastric instillation of vehicle (Tris buffer) (A), indomethacin alone (B) or indomethacin with previous hind limb ischemic preconditioning $(\mathrm{C})$. The arrows indicate the linear gastric lesions. 

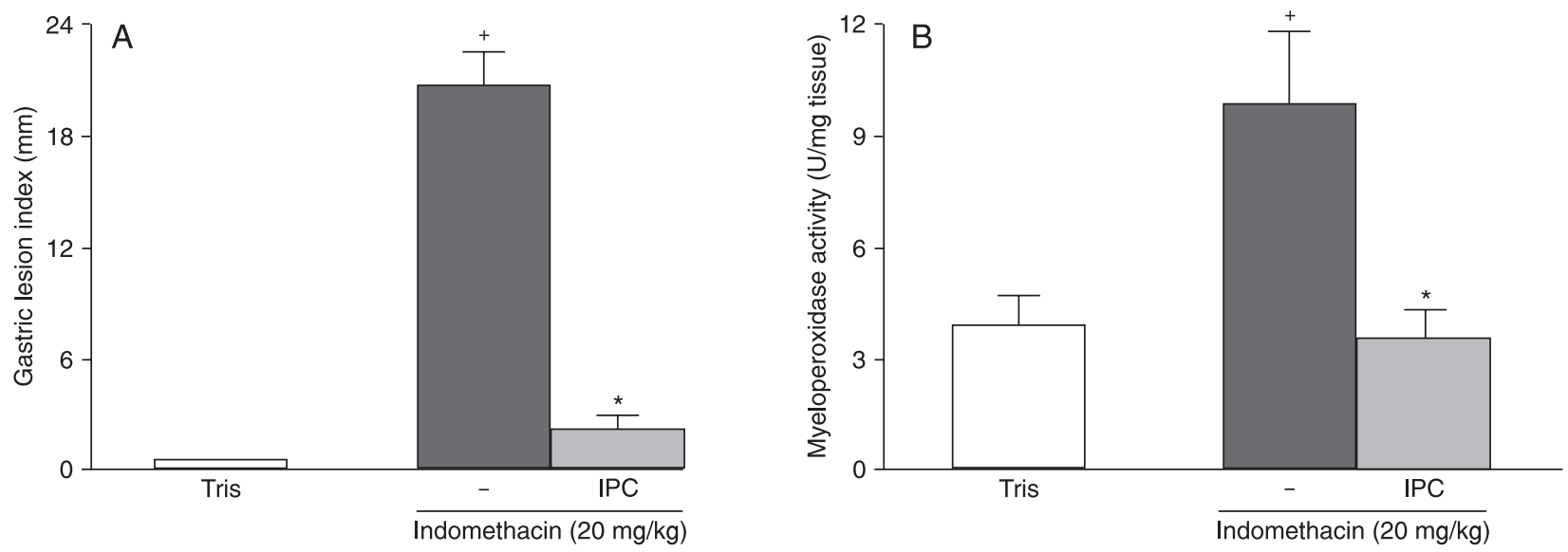

Figure 7. Protective effect of rat hind limb ischemic preconditioning on gastric injury (A) and gastric neutrophil accumulation (B) induced by indomethacin. $A$, The column heights indicate the gastric lesion index after Tris buffer (Tris), after only indomethacin (-), and after indomethacin plus ischemic preconditioning (IPC). $B$, The column heights indicate the gastric myeloperoxidase activity after Tris buffer (Tris), after only indomethacin (-), and after indomethacin plus ischemic preconditioning (IPC). Data are reported as means \pm SEM. ${ }^{*} \mathrm{P}<0.05$ compared to indomethacin; ${ }^{+} \mathrm{P}<0.05$ compared to Tris (ANOVA, followed by the Tukey test).

7B shows that neutrophil migration in the gastric mucosa was significantly greater $3 \mathrm{~h}$ after INDO administration, an effect that was also inhibited by right hind limb IPC.

\section{Discussion}

In this study, we determined the local and systemic effects of IPC on a rat model of acute inflammation. IPC of the right hind limb decreased inflammation not only in the same limb, but also in the contralateral limb and in distant organs, such as the bladder and stomach.

The present results suggest that IPC has an antiinflammatory effect on $\mathrm{Cg}$ - and Dx-induced paw edema, since hind limb IPC inhibited the edema and changes in vascular permeability induced by these substances. This protective effect was observed in both limbs, regardless of the limb used for IPC.

$\mathrm{Cg}$ and Dx have been shown to increase vascular permeability by different mechanisms. While Dx induces fluid accumulation due to mast cell degranulation with little protein and few neutrophils, $\mathrm{Cg}$ induces a protein-rich exudate containing a large number of neutrophils (23). Since IPC inhibited Cg-and Dx-induced edema, it may be assumed that IPC reduces both polymorphonuclear leukocyte-dependent and mast cell-dependent edema.

Cg-induced neutrophil migration depends on the release of chemotactic mediators by resident cells (24). Thus, it is possible that the protective mechanism of IPC in Cg-induced polymorphonuclear leukocyte-dependent edema involves the inhibition of macrophage and/or mast cell-related release of chemotactic agents such as tumor necrosis factor alpha (TNF- $\alpha$ ) and interleukin (IL)-1. This notion is supported by the fact that IPC has been shown to be associated with reduced levels of TNF- $\alpha$, IL-1 and IL-6 in cardiac IRI (25). In addition, the cardioprotective and antiarrhythmic effects of IPC have been ascribed to the degranulation of cardiac mast cells (26). Depletion of cytotoxic mediators during IPC, and the consequent reduced availability of such mediators during inflammatory events, may be associated with greater preservation of structures in target organs. In fact, it was recently demonstrated in a simple model that IPC with brief forearm ischemia suppresses the proinflammatory gene expression in human circulating leukocytes (10).

TNF- $\alpha$ has been implicated in the development of ischemic tolerance, since this effect is not observed in TNF- $\alpha$ knockout mice (27). Likewise, in one study, TNF-a protected cultured neurons against ischemic death and cultured astrocytes against the proinflammatory effects of TNF- $\alpha$ (28). The protective effect of IPC on $\mathrm{Cg}$ - and Dx-induced paw edema may actually be due to the release of preformed TNF- $\alpha$ by resident cells during limb reperfusion.

Endogenous NO is another mediator involved in the inflammatory effect of $\mathrm{Cg}(29)$ and in the protective IPC phenomenon. Kawahara et al. (30) suggested that NO produced during ischemia was fundamentally toxic, but critical to the development of neuronal tolerance. lalenti et al. (31) demonstrated that NO donors significantly inhibited Cg-induced leukocyte infiltration while high doses of NO donors increased leukocyte accumulation. Thus, it may be suggested that IPC inhibits the effect of $\mathrm{Cg}$ by stimulating the production of small amounts of NO prior to the inflammatory challenge.

Hemorrhagic cystitis is currently a limiting side effect observed in patients submitted to cyclophosphamide- or IFO-based cancer chemotherapy regimens (18). In the absence of adequate uroprotection, $\mathrm{HC}$ becomes dose- 
limiting, with an average incidence of $40 \%$. Such toxicity is attributed to the renal excretion of acrolein, a urotoxic metabolite of IFO. It has been proposed that urothelial damage occurs by direct contact with acrolein, which causes edema, ulceration, neovascularization, hemorrhage, and necrosis (19). Our findings for IFO-induced $\mathrm{HC}$ in rats show that hind limb rIPC attenuates both vesical edema and increased vascular permeability in this inflammatory condition.

IPC of the bladder is technically complex because of the rich vascular anastomoses and collateral circulation of the organ. Few studies have reported protective effects of IPC on the bladder (32), but rIPC, as described in the present study, appears to be a promising strategy in this line of research.

Although cyclophosphamide- and IFO-induced urothelial damage is believed to occur through direct contact with acrolein, the role of endogenous inflammatory mediators in the mechanism of bladder injury remains unclear (19). Important mechanisms involved in the pathogenesis of experimental cyclophosphamide and IFO-induced $\mathrm{HC}$ have been recently clarified. Thus, cytokines such as TNF- $\alpha$ and IL-1 were shown to be crucial mediators involved in cystitis as well as in urothelial damage and hemorrhage $(20,33)$. Furthermore, NO was shown to be the final mediator of urothelial damage and hemorrhage in cystitis since drugs inhibiting NO synthesis reduced edema and hemorrhage in a dose-dependent manner (16). The administration of anti-TNF- $\alpha$ or anti-IL-1 $\beta$ serum significantly decreased cyclophosphamide and IFO-induced vesical inflammation and reverted increased inducible NO synthase expression and activity $(16,33)$. Moreover, the induction of NO synthase in the inflamed bladder appears to require the presence of platelet-activating factor (16).

The protective effect of hind limb rIPC on IFO-induced $\mathrm{HC}$ may be explained by the inhibitory effect of IFO-induced release of inflammatory mediators such as TNF-a, IL-1 and platelet-activating factor from macrophages and/or mast cells. These would reduce NO synthase induction in the inflamed bladder and the consequent increase in NO production. In addition, rIPC possibly inhibits IFO-induced inducible NO synthase by producing small amounts of $\mathrm{NO}$ and/or TNF- $\alpha$ during the reperfusion phase of IPC, especially since production of these substances has been implicated in the development of ischemic tolerance after induction of IPC $(27,28,30)$.

Nonsteroidal anti-inflammatory drugs (NSAIDs) are among the most widely used drugs in the world (34). The main side effect of NSAIDs is gastric injury, the mechanism of which is generally believed to be related to the ability of these agents to inhibit gastric prostaglandin (PG) generation (34). However, recently published evidence suggests that leukocyte adherence to the vascular endothelium (35), microcirculatory disturbances, superoxide radicals, and protease release play a relevant pathogenic role in NSAID- induced gastropathy (36). In the present study, hind limb rIPC significantly reduced mucosal lesions and neutrophil infiltration in INDO-induced gastric injury in rats.

Gastric IPC achieved with short episodes of celiac artery occlusion attenuated reduced gastric blood flow rates, PGE2 generation and the numerous gastric lesions caused by prolonged gastric IRI. This protective effect was associated with $\mathrm{mRNA}$ - and protein-related overexpression of cyclooxygenase-2 (COX-2) in the preconditioned gastric mucosa, while COX-1 mRNA remained unchanged, supporting the notion that endogenous PG produced in excessive amounts by COX-2 plays an important role in the mechanism of gastric preconditioning (37). Thus, hind limb rIPC possibly inhibits the effects of INDO on the stomach by overexpression of COX-2 in the gastric mucosa. Accordingly, Brzozowski et al. (38) recently reported that IPC of remote organs, such as the heart and liver, inhibited gastric IRI by increasing gastric blood flow and PGE2 generation.

Souza et al. (39) recently reported a reduction in INDOinduced gastric injury and granulocyte infiltration in TNF receptor 1-or inducible NO synthase-deficient mice, thereby clearly demonstrating the importance of these mediators in inflammation. In this context, the gastroprotective effect of hind limb rIPC may involve the inhibition of TNF- $\alpha$ or NO production. The production of small amounts of $\mathrm{NO}$ and/ or TNF- $\alpha$ during the reperfusion phase of IPC can inhibit INDO-induced production of large amounts of $\mathrm{NO}$ and/or TNF- $\alpha$ in the gastric mucosa.

The local and systemic inflammatory response associated with IRI may involve the ROS-generating system, xanthine oxidase (XOD). During ischemia, xanthine dehydrogenase $(\mathrm{XDH})$, the physiologic form of the enzyme, is converted to the oxygen radical-producing form, XOD. Concurrently, there is an accumulation of xanthine, the substrate for XOD. Upon reoxygenation, XOD reacts with molecular oxygen to produce ROS. It is well known that ROS initiates a cascade of deleterious cellular responses leading to inflammation, cell death, and organ failure. On the other hand, the injurious effects of xanthine/XOD are not limited to the area submitted to ischemia. Xanthine/ $\mathrm{XOD}$ is released into the circulation during reperfusion of the ischemic tissue. Thus, this ROS generation system plays an essential role in the pathogenesis of systemic complications of IRI, including neutrophil infiltration and oxidant stress in the lung (40).

Fernández et al. (40) reported that IPC conferred protection against both liver and lung damage following liver transplantation. This endogenous protective mechanism was capable of blocking xanthine/XOD generation in liver grafts during cold ischemia and ROS generation, neutrophil infiltration and oxidant stress in the lung. Thus, the antiinflammatory effect of hind limb rIPC at distant sites, such as contralateral limb, bladder and stomach, may be due to reduction of conversion of $\mathrm{XDH}$ to XOD, ROS generation, neutrophil infiltration, and oxidant stress at these sites. 
Adenosine is an extracellular molecule that is both a trigger and a mediator of IPC, as demonstrated in previous studies. Adenosine production occurs in myocytes, endothelial cells, and vascular cells. During ischemia the imbalance between oxygen supply and demand results in net breakdown of adenosine triphosphate (ATP) and release of adenosine, which can increase up to 50 -fold (1).

Adenosine is cytoprotective and has an important role in protection from IRI by direct IPC of the heart and liver through the adenosine receptors $\mathrm{A} 1$ and $\mathrm{A} 3$ in the heart and $\mathrm{A} 2 \mathrm{~A}$ in the liver. Adenosine is also released after direct IPC of skeletal muscles as a physiological response in order to reduce injury to the microcirculation, and brief hind limb ischemia by the release of adenosine may protect remote organs against IRI (6).

Released adenosine has been shown to be an effector molecule in skeletal muscle IPC. Remote IPC increased plasma adenosine levels and the protective effect was partially blocked with reserpine. Prior adenosine blockade did not completely abolish latissmus dorsi flap protection by rIPC (limb ischemia, $3 \times 10$-min cycles); however, adenosine blockade with 8 (p-sulfophenyl)theophylline and the free radical scavenger mercaptopropionyl glycine completely

\section{References}

1. Tapuria N, Kumar Y, Habib MM, Abu AM, Seifalian AM, Davidson $B R$. Remote ischemic preconditioning: a novel protective method from ischemia reperfusion injury - a review. J Surg Res 2008; 150: 304-330.

2. Zelenock GB, D'Alecy LG, Fantoni JC III, Shlafer M, Stanley JC. Clinical ischemic syndromes: mechanism and consequences of tissue injury. St. Louis: CV Mosby; 1990.

3. Kadambi A, Skalak TC. Role of leukocytes and tissuederived oxidants in short-term skeletal muscle ischemiareperfusion injury. Am J Physiol Heart Circ Physiol 2000; 278: $\mathrm{H} 435-\mathrm{H} 443$.

4. Marber MS, Latchman DS, Walker JM, Yellon DM. Cardiac stress protein elevation 24 hours after brief ischemia or heat stress is associated with resistance to myocardial infarction. Circulation 1993; 88: 1264-1272.

5. Kharbanda RK, Mortensen UM, White PA, Kristiansen SB, Schmidt MR, Hoschtitzky JA, et al. Transient limb ischemia induces remote ischemic preconditioning in vivo. Circulation 2002; 106: 2881-2883.

6. Kanoria S, Jalan R, Seifalian AM, Williams R, Davidson BR. Protocols and mechanisms for remote ischemic preconditioning: a novel method for reducing ischemia reperfusion injury. Transplantation 2007; 84: 445-458.

7. Downey JM, Davis AM, Cohen MV. Signaling pathways in ischemic preconditioning. Heart Fail Rev 2007; 12: 181188.

8. Downey JM, Krieg T, Cohen MV. Mapping preconditioning's signaling pathways: an engineering approach. Ann N Y Acad Sci 2008; 1123: 187-196.

9. Fessler MB, Malcolm KC, Duncan MW, Worthen GS. A genomic and proteomic analysis of activation of the human abolished the rIPC effect, suggesting that adenosine plays a partial role in rIPC (1)

The effects of adenosine include vasodilatation, inhibition of leukocyte adhesion, neutrophil and platelet function, and free radical production (1). From this perspective, the anti-inflammatory effect of rIPC observed in our study may be due to adenosine release after brief hind limb ischemia, which may protect remote organs against different inflammatory challenges.

Our results suggest that hind limb IPC has a local and systemic protective effect on acute inflammatory conditions. Although the mechanisms of distant protection are not completely understood, they may include a potent and broad-spectrum suppression of inflammatory signals. The clinical application of this mechanism may make an important contribution in conditions in which the inflammatory injury has a deleterious effect.

\section{Acknowledgments}

The authors gratefully acknowledge the technical assistance of Maria Silvandira Freire França (Universidade Federal do Ceará, Fortaleza, CE, Brazil). neutrophil by lipopolysaccharide and its mediation by $\mathrm{p} 38$ mitogen-activated protein kinase. J Biol Chem 2002; 277: 31291-31302.

10. Konstantinov IE, Arab S, Kharbanda RK, Li J, Cheung MM, Cherepanov V, et al. The remote ischemic preconditioning stimulus modifies inflammatory gene expression in humans. Physiol Genomics 2004; 19: 143-150.

11. Salehipour M, Khezri A, Monabbati A, Jalaeian $H$, Kroup $\mathrm{M}$, Azizi $\mathrm{V}$, et al. Ischemic preconditioning protects the dog kidney from ischemia-reperfusion injury. Urol Int 2007; 79: 328-331.

12. Harkin DW, Barros D'Sa AA, McCallion K, Hoper M, Campbell FC. Ischemic preconditioning before lower limb ischemia-reperfusion protects against acute lung injury. $J$ Vasc Surg 2002; 35: 1264-1273.

13. Kuntscher MV, Schirmbeck EU, Menke H, Klar E, Gebhard MM, Germann G. Ischemic preconditioning by brief extremity ischemia before flap ischemia in a rat model. Plast Reconstr Surg 2002; 109: 2398-2404.

14. Landucci EC, Antunes E, Donato JL, Faro R, Hyslop S, Marangoni $S$, et al. Inhibition of carrageenin-induced rat paw oedema by crotapotin, a polypeptide complexed with phospholipase A2. Br J Pharmacol 1995; 114: 578-583.

15. Leme JG, Wilhelm DL. The effects of adrenalectomy and corticosterone on vascular permeability responses in the skin of the rat. Br J Exp Pathol 1975; 56: 402-407.

16. Souza-Fiho MV, Lima MV, Pompeu MM, Ballejo G, Cunha $F Q$, Ribeiro RA. Involvement of nitric oxide in the pathogenesis of cyclophosphamide-induced hemorrhagic cystitis. Am J Pathol 1997; 150: 247-256.

17. Vieira MM, Brito GA, Belarmino-Filho JN, Macedo FY, Nery 
$E A$, Cunha FQ, et al. Use of dexamethasone with mesna for the prevention of ifosfamide-induced hemorrhagic cystitis. Int J Urol 2003; 10: 595-602.

18. Philips FS, Sternberg SS, Cronin AP, Vidal PM. Cyclophosphamide and urinary bladder toxicity. Cancer Res 1961; 21: 1577-1589.

19. Cox PJ. Cyclophosphamide cystitis - identification of acrolein as the causative agent. Biochem Pharmacol 1979; 28: 2045-2049.

20. Gomes TN, Santos CC, Souza-Filho MV, Cunha FQ, Ribeiro RA. Participation of TNF-alpha and IL-1 in the pathogenesis of cyclophosphamide-induced hemorrhagic cystitis. Braz J Med Biol Res 1995; 28: 1103-1108.

21. Santucci L, Fiorucci S, Giansanti M, Brunori PM, Di Matteo FM, Morelli A. Pentoxifylline prevents indomethacin induced acute gastric mucosal damage in rats: role of tumour necrosis factor alpha. Gut 1994; 35: 909-915.

22. Bradley PP, Christensen RD, Rothstein G. Cellular and extracellular myeloperoxidase in pyogenic inflammation. Blood 1982; 60: 618-622.

23. Lo TN, Almeida AP, Beaven MA. Dextran and carrageenan evoke different inflammatory responses in rat with respect to composition of infiltrates and effect of indomethacin. $J$ Pharmacol Exp Ther 1982; 221: 261-267.

24. de Souza GE, Ferreira SH. Blockade by antimacrophage serum of the migration of PMN neutrophils into the inflamed peritoneal cavity. Agents Actions 1985; 17: 97-103.

25. Hiasa G, Hamada M, Ikeda S, Hiwada K. Ischemic preconditioning and lipopolysaccharide attenuate nuclear factorkappaB activation and gene expression of inflammatory cytokines in the ischemia-reperfused rat heart. Jpn Circ $J$ 2001; 65: 984-990.

26. Parikh V, Singh M. Possible role of cardiac mast cell degranulation and preservation of nitric oxide release in isolated rat heart subjected to ischaemic preconditioning. Mol Cell Biochem 1999; 199: 1-6.

27. Smith RM, Suleman N, McCarthy J, Sack MN. Classic ischemic but not pharmacologic preconditioning is abrogated following genetic ablation of the TNFalpha gene. Cardiovasc Res 2002; 55: 553-560.

28. Ginis I, Jaiswal R, Klimanis D, Liu J, Greenspon J, Hallenbeck JM. TNF-alpha-induced tolerance to ischemic injury involves differential control of NF-kappaB transactivation: the role of NF-kappaB association with p300 adaptor. $J$ Cereb Blood Flow Metab 2002; 22: 142-152.

29. Sautebin L, lalenti A, lanaro A, Di Rosa M. Endogenous nitric oxide increases prostaglandin biosynthesis in carrageenin rat paw oedema. Eur J Pharmacol 1995; 286: 219-222.

30. Kawahara K, Yanoma J, Tanaka M, Nakajima T, Kosugi T. Nitric oxide produced during ischemia is toxic but crucial to preconditioning-induced ischemic tolerance of neurons in culture. Neurochem Res 2004; 29: 797-804.

31. Ialenti A, lanaro A, Maffia P, Sautebin L, Di Rosa M. Nitric oxide inhibits leucocyte migration in carrageenin-induced rat pleurisy. Inflamm Res 2000; 49: 411-417.

32. Lorenzi B, McMurray G, Jarvis G, Brading AF. Preconditioning protects the guinea-pig urinary bladder against ischaemic conditions in vitro. Neurourol Urodyn 2003; 22: 687-692.

33. Ribeiro RA, Freitas HC, Campos MC, Santos CC, Figueiredo FC, Brito GA, et al. Tumor necrosis factor-alpha and interleukin-1beta mediate the production of nitric oxide involved in the pathogenesis of ifosfamide induced hemorrhagic cystitis in mice. J Urol 2002; 167: 2229-2234.

34. Vane JR. Inhibition of prostaglandin synthesis as a mechanism of action for aspirin-like drugs. Nat New Biol 1971; 231: 232-235.

35. Asako H, Kubes P, Wallace J, Wolf RE, Granger DN. Modulation of leukocyte adhesion in rat mesenteric venules by aspirin and salicylate. Gastroenterology 1992; 103: 146152.

36. Takeuchi K, Takehara K, Ohuchi T. Diethyldithiocarbamate, a superoxide dismutase inhibitor, reduces indomethacin-induced gastric lesions in rats. Digestion 1996; 57: 201-209.

37. Pajdo R, Brzozowski T, Konturek PC, Kwiecien S, Konturek SJ, Sliwowski Z, et al. Ischemic preconditioning, the most effective gastroprotective intervention: involvement of prostaglandins, nitric oxide, adenosine and sensory nerves. Eur J Pharmacol 2001; 427: 263-276.

38. Brzozowski T, Konturek PC, Konturek SJ, Pajdo R, Kwiecien $\mathrm{S}$, Pawlik $\mathrm{M}$, et al. Ischemic preconditioning of remote organs attenuates gastric ischemia-reperfusion injury through involvement of prostaglandins and sensory nerves. Eur $J$ Pharmacol 2004; 499: 201-213.

39. Souza MH, Lemos HP, Oliveira RB, Cunha FQ. Gastric damage and granulocyte infiltration induced by indomethacin in tumour necrosis factor receptor 1 (TNF-R1) or inducible nitric oxide synthase (iNOS) deficient mice. Gut 2004; 53: 791-796.

40. Fernandez L, Heredia N, Grande L, Gomez G, Rimola A, Marco A, et al. Preconditioning protects liver and lung damage in rat liver transplantation: role of xanthine/xanthine oxidase. Hepatology 2002; 36: 562-572. 